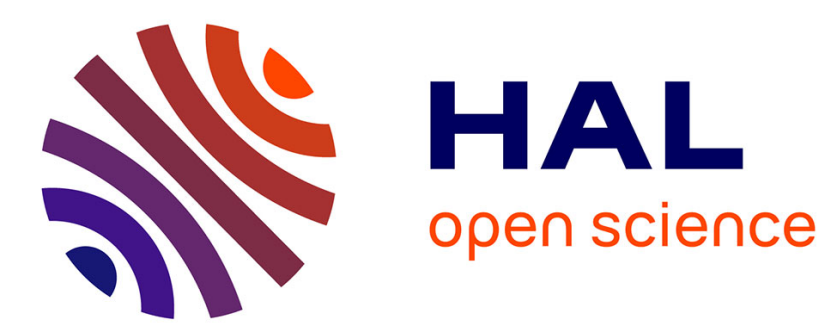

\title{
ON THE RADICALS OF A GROUP THAT DOES NOT HAVE THE INDEPENDENCE PROPERTY
}

\author{
Cédric Milliet
}

\section{To cite this version:}

Cédric Milliet. ON THE RADICALS OF A GROUP THAT DOES NOT HAVE THE INDEPENDENCE PROPERTY. The Journal of Symbolic Logic, 2016, 81 (4), pp.1444-1450. 10.1017/jsl.2015.56 . hal-01256813

\section{HAL Id: hal-01256813 \\ https://hal.science/hal-01256813}

Submitted on 15 Jan 2016

HAL is a multi-disciplinary open access archive for the deposit and dissemination of scientific research documents, whether they are published or not. The documents may come from teaching and research institutions in France or abroad, or from public or private research centers.
L'archive ouverte pluridisciplinaire HAL, est destinée au dépôt et à la diffusion de documents scientifiques de niveau recherche, publiés ou non, émanant des établissements d'enseignement et de recherche français ou étrangers, des laboratoires publics ou privés. 


\title{
ON THE RADICALS OF A GROUP THAT DOES NOT HAVE THE INDEPENDENCE PROPERTY
}

\author{
CÉDRIC MILLIET
}

\begin{abstract}
We give an example of a pure group that does not have the independence property, whose Fitting subgroup is neither nilpotent nor definable and whose soluble radical is neither soluble nor definable. This answers a question asked by E. Jaligot in May 2013.
\end{abstract}

The Fitting subgroup of a stable group is nilpotent and definable (F. Wagner [Wag95]). More generally, the Fitting subgroup of a group that satisfies the descending chain condition on centralisers is nilpotent (J. Derakhshan, F. Wagner [DW97]) and definable (F. Wagner [Wag99, Corollary 2.5], see also [OH13] and [AB14]). The soluble radical of a superstable group is soluble and definable (A. Baudish [Bau90]). Whether this also holds for a stable group is still an open question.

Inspired by [MT12], we provide an example of a pure group that does not have the independence property, whose Fitting subgroup is neither nilpotent nor definable and whose soluble radical is neither soluble nor definable. The proofs require some algebra because we have decided to provide a precise computation of the Fitting subgroup and soluble radical of the group considered.

Definition 1 (independence property). Let $M$ be a structure. A formula $\varphi(x, y)$ has the independence property in $M$ if for all $n \in \omega$, there are tuples $a_{1}, \ldots, a_{n}$ and $\left(b_{J}\right)_{J \subset\{1, \ldots, n\}}$ of $M$ such that $\left(M \models \varphi\left(a_{i}, b_{J}\right)\right) \Longleftrightarrow i \in J$. M does not have the independence property (or is NIP for short) if no formula has the independence property in $M$.

Let $\mathbf{L}$ be a first order language, $M$ an $\mathbf{L}$-structure. A set $X$ is interpretable in $M$ if there is a definable subset $Y \subset M^{n}$ in $M$ and a definable equivalence relation $E$ on $X$ such that $X=Y / E$. A family $\left\{Y_{i} / E_{i}: i \in I\right\}$ of interpretable sets in $M$ is uniformly interpretable in $M$ if the corresponding families $\left\{Y_{i}: i \in I\right\}$ and $\left\{E_{i}: i \in I\right\}$ are uniformly definable in $M$.

Let $L$ be yet another first order language. An $L$-structure $N$ is interpretable in $M$ if its domain, functions, relations and constants are interpretable sets in $M$. A family of $L$ structures $\left\{N_{i}: i \in I\right\}$ is uniformly interpretable in $M$ if the family of domains is uniformly interpretable in $M$, as well as, for each symbol $s$ of the language $L$, the family $\left\{s_{i}: i \in I\right\}$ of interpretations of $s$ in $N_{i}$.

Lemma 2 (D. Macpherson, K. Tent [MT12]). Let $M$ be an L-structure that does not have the independence property and let $\left\{N_{i}: i \in I\right\}$ be a family of L-structures that is uniformly interpretable in $M$. For every ultrafilter $\mathcal{U}$ on $I$, the $L$-structure $\prod_{i \in I} N_{i} / \mathcal{U}$ does not have the independence property.

Key words and phrases. Model theory; independence property; Fitting subgroup and soluble radical; ultraproducts.

Many thanks to T. Altinel and A. Fehm for stimulating conversations, as well as to S. Kulhmann. 
Corollary 3. Let $m$ and $n$ be natural numbers and $p$ a prime number. Let us consider the general linear group $\mathrm{GL}_{\mathrm{m}}\left(\mathbf{Z} / p^{n} \mathbf{Z}\right)$ over the finite ring $\mathbf{Z} / p^{n} \mathbf{Z}$. Let $\mathcal{U}$ be an ultrafilter on $\mathbf{N}$ and let $G$ be the ultraproduct

$$
G=\prod_{n \in \mathbf{N}} \mathrm{GL}_{\mathrm{m}}\left(\mathbf{Z} / p^{n} \mathbf{Z}\right) / \mathcal{U}
$$

The pure group $G$ does not have the independence property.

Proof. Consider the group $\mathrm{GL}_{\mathrm{m}}\left(\mathbf{Z}_{p}\right)$ over the ring $\mathbf{Z}_{p}$ of $p$-adic integers, and the normal subgroups $\mathbf{1}+p^{n} \mathrm{M}_{\mathrm{m}}\left(\mathbf{Z}_{p}\right)$ for every $n \geqslant 1$. One has the group isomorphism

$$
\mathrm{GL}_{\mathrm{m}}\left(\mathbf{Z} / p^{n} \mathbf{Z}\right) \simeq \mathrm{GL}_{\mathrm{m}}\left(\mathbf{Z}_{p}\right) / \mathbf{1}+p^{n} \mathrm{M}_{\mathrm{m}}\left(\mathbf{Z}_{p}\right) .
$$

Therefore, the family of groups $\left\{\mathrm{GL}_{\mathrm{m}}\left(\mathbf{Z} / p^{n} \mathbf{Z}\right): n \in \mathbf{N}\right\}$ is uniformly interpretable in the ring $\mathrm{M}_{\mathrm{m}}\left(\mathbf{Q}_{p}\right)$, which is interpretable in the field $\mathbf{Q}_{p}$ of $p$-adic numbers, hence NIP by [Mat93]. By Lemma 2, the group $G$ does not have the independence property.

\section{Preliminaries on the normal structure of $\mathrm{GL}_{\mathrm{m}}\left(\mathbf{Z} / p^{n} \mathbf{Z}\right)$}

Given a field $k$, the normal subgroups of the general linear group $\mathrm{GL}_{\mathrm{m}}(k)$ are precisely the subgroups of the centre and the subgroups containing the special linear group $\mathrm{SL}_{\mathrm{m}}(k)$ (J. Dieudonné [Die55]). In particular, the maximal normal soluble subgroup of $\mathrm{GL}_{\mathrm{m}}(k)$ is the centre, except for the two soluble groups $\mathrm{GL}_{2}\left(\mathbf{F}_{2}\right)$ and $\mathrm{GL}_{2}\left(\mathbf{F}_{3}\right)$. The situtation is different for the general linear group $\mathrm{GL}_{\mathrm{m}}\left(\mathbf{Z} / p^{n} \mathbf{Z}\right)$ over the $\operatorname{ring} \mathbf{Z} / p^{n} \mathbf{Z}$, whose normal subgroups are classified by J. Brenner [Bre38]. We follow also W. Klingenberg [Kli60] who deals with the normal subgroups of the general linear group over a local ring $R$, which applies in particular to $\mathbf{Z} / p^{n} \mathbf{Z}$.

The centre of $\mathrm{GL}_{\mathrm{m}}\left(\mathbf{Z} / p^{n} \mathbf{Z}\right)$ is the subgroup of homotheties $\left(\mathbf{Z} / p^{n} \mathbf{Z}\right)^{\times} \cdot \mathbf{1}$. The general congruence subgroup of $\mathrm{GL}_{\mathrm{m}}\left(\mathbf{Z} / p^{n} \mathbf{Z}\right)$ of order $\ell$ is

$$
\mathrm{GC}_{\mathrm{m}}(\ell)=\left(\mathbf{Z} / p^{n} \mathbf{Z}\right)^{\times} \cdot \mathbf{1}+p^{\ell} \mathrm{M}_{\mathrm{m}}\left(\mathbf{Z} / p^{n} \mathbf{Z}\right) .
$$

It is a normal subgroup of $\mathrm{GL}_{\mathrm{m}}\left(\mathbf{Z} / p^{n} \mathbf{Z}\right)$. For every element $g$ of $\mathrm{GL}_{\mathrm{m}}\left(\mathbf{Z} / p^{n} \mathbf{Z}\right)$, there is a maximal $\ell \leqslant n$ such that $g$ belongs to $\mathrm{GC}_{\mathrm{m}}(\ell)$. We call $\ell$ the congruence order of $g$.

The special linear subgroup of $\mathrm{GL}_{\mathrm{m}}\left(\mathbf{Z} / p^{n} \mathbf{Z}\right)$ of matrices having determinant 1 is written $\mathrm{SL}_{\mathrm{m}}\left(\mathbf{Z} / p^{n} \mathbf{Z}\right)$. An elementary transvection is an element of $\mathrm{SL}_{\mathrm{m}}\left(\mathbf{Z} / p^{n} \mathbf{Z}\right)$ of the form $\mathbf{1}+r e_{i j}$ for $r \in \mathbf{Z} / p^{n} \mathbf{Z}$ and $i \neq j$. A transvection is a conjugate of an elementary transvection.

Proposition 4 (J. Brenner [Bre38, Theorem 1.5]). Let $\tau$ a transvection of congruence order $\ell$. The normal subgroup of $\mathrm{GL}_{\mathrm{m}}\left(\mathbf{Z} / p^{n} \mathbf{Z}\right)$ generated by $\tau$ is

$$
\left\langle\tau^{\mathrm{GL}}\left(\mathbf{Z} / p^{n} \mathbf{Z}\right)\right\rangle=\mathrm{SL}_{\mathrm{m}}\left(\mathbf{Z} / p^{n} \mathbf{Z}\right) \cap\left(\mathbf{1}+p^{\ell} \mathrm{M}_{\mathrm{m}}\left(\mathbf{Z} / p^{n} \mathbf{Z}\right)\right) .
$$

Theorem 5 (J. Brenner [Bre38]). Let $m p \geqslant 6$ and $g$ an element of $\mathrm{GL}_{\mathrm{m}}\left(\mathbf{Z} / p^{n} \mathbf{Z}\right)$ of congruence order $\ell$. The normal subgroup $\left\langle g^{\mathrm{GL}_{\mathrm{m}}\left(\mathbf{Z} / p^{n} \mathbf{Z}\right)}\right\rangle$ of $\mathrm{GL}_{\mathrm{m}}\left(\mathbf{Z} / p^{n} \mathbf{Z}\right)$ generated by $g$ satisfies

$$
\mathrm{SL}_{\mathrm{m}}\left(\mathbf{Z} / p^{n} \mathbf{Z}\right) \cap\left(\mathbf{1}+p^{\ell} \mathrm{M}_{\mathrm{m}}\left(\mathbf{Z} / p^{n} \mathbf{Z}\right)\right) \subset\left\langle g^{\mathrm{GL}_{\mathrm{m}}\left(\mathbf{Z} / p^{n} \mathbf{Z}\right)}\right\rangle \subset\left(\mathbf{Z} / p^{n} \mathbf{Z}\right)^{\times} \cdot \mathbf{1}+p^{\ell} \mathrm{M}_{\mathrm{m}}\left(\mathbf{Z} / p^{n} \mathbf{Z}\right) .
$$

For any real number $x$, we write $\lfloor x\rfloor$ for the floor of $x$, that is $\lfloor x\rfloor$ is the greatest integer $k$ such that $k \leqslant x$. 
Lemma 6. For any $m \geqslant 2, \ell \geqslant 1$ and $n \geqslant 1$, the group $\mathbf{1}+p^{\ell} \mathrm{M}_{\mathrm{m}}\left(\mathbf{Z} / p^{n} \mathbf{Z}\right)$ is a normal nilpotent subgroup of $\mathrm{GL}_{\mathrm{m}}\left(\mathbf{Z} / p^{n} \mathbf{Z}\right)$ of nilpotency class $\left\lfloor\frac{n-1}{\ell}\right\rfloor$.

Proof. For every $x$ in $\mathrm{M}_{\mathrm{m}}\left(\mathbf{Z} / p^{n} \mathbf{Z}\right)$, one has

$$
(\mathbf{1}+p x)^{p}=\mathbf{1}+\sum_{k=1}^{p}(p x)^{k} C_{p}^{k}=\mathbf{1}+p^{2} y .
$$

It follows that $\mathbf{1}+p \mathrm{M}_{\mathrm{m}}\left(\mathbf{Z} / p^{n} \mathbf{Z}\right)$ is a nilpotent $p$-group. Its iterated centres are

$$
\begin{aligned}
Z\left(H_{n}\right) & =\left(1+p \mathbf{Z} / p^{n} \mathbf{Z}\right) \cdot \mathbf{1}+p^{n-1} \mathrm{M}_{\mathrm{m}}\left(\mathbf{Z} / p^{n} \mathbf{Z}\right) \\
Z_{2}\left(H_{n}\right) & =\left(1+p \mathbf{Z} / p^{n} \mathbf{Z}\right) \cdot \mathbf{1}+p^{n-2} \mathrm{M}_{\mathrm{m}}\left(\mathbf{Z} / p^{n} \mathbf{Z}\right) \\
\vdots & \\
Z_{n-2}\left(H_{n}\right) & =\left(1+p \mathbf{Z} / p^{n} \mathbf{Z}\right) \cdot \mathbf{1}+p^{2} \mathrm{M}_{\mathrm{m}}\left(\mathbf{Z} / p^{n} \mathbf{Z}\right) \\
Z_{n-1}\left(H_{n}\right) & =\mathbf{1}+p \mathrm{M}_{\mathrm{m}}\left(\mathbf{Z} / p^{n} \mathbf{Z}\right),
\end{aligned}
$$

so the nilpotency class of $\mathbf{1}+p \mathrm{M}_{\mathrm{m}}\left(\mathbf{Z} / p^{n} \mathbf{Z}\right)$ is $n-1$ when $n \geqslant 1$. For every natural number $q$ satisfying $n-q \ell \geqslant \ell$, one has

$$
Z_{q}\left(\mathbf{1}+p^{\ell} \mathrm{M}_{\mathrm{m}}\left(\mathbf{Z} / p^{n} \mathbf{Z}\right)\right)=\left(1+p^{\ell} \mathbf{Z} / p^{n} \mathbf{Z}\right) \cdot \mathbf{1}+p^{n-q \ell} \mathrm{M}_{\mathrm{m}}\left(\mathbf{Z} / p^{n} \mathbf{Z}\right),
$$

so the greatest $q$ such that the above $q$ th centre is a proper subgroup is the greatest $q$ satisfying $n-q \ell>\ell$. As one has

$$
n-q \ell>\ell \Longleftrightarrow n-1-q \ell \geqslant \ell \Longleftrightarrow q \leqslant \frac{n-1}{\ell}-1 \text {, }
$$

this greatest $q$ is precisely $\left\lfloor\frac{n-1}{\ell}\right\rfloor-1$.

For any real number $x$, we write $\lceil x\rceil$ for the ceiling of $x$, that is $\lceil x\rceil$ is the least integer $k$ such that $k \geqslant x$.

Lemma 7. For any $1 \leqslant \ell \leqslant n$ and $m \geqslant 3$, the group $\mathbf{1}+p^{\ell} \mathrm{M}_{\mathrm{m}}\left(\mathbf{Z} / p^{n} \mathbf{Z}\right)$ is soluble of derived length $\left\lceil\log _{2} \frac{n}{\ell}\right\rceil$.

Proof. Let us write $\mathrm{PC}_{\mathrm{m}}(\ell)=\mathbf{1}+p^{\ell} \mathrm{M}_{\mathrm{m}}\left(\mathbf{Z} / p^{n} \mathbf{Z}\right)$ and show that

$$
\left(\mathrm{SL}_{\mathrm{m}}\left(\mathbf{Z} / p^{n} \mathbf{Z}\right) \cap \mathrm{PC}_{\mathrm{m}}(\ell)\right)^{\prime}=\mathrm{PC}_{\mathrm{m}}(\ell)^{\prime}=\mathrm{SL}_{\mathrm{m}}\left(\mathbf{Z} / p^{n} \mathbf{Z}\right) \cap \mathrm{PC}_{\mathrm{m}}(2 \ell) .
$$

Let $\alpha=\mathbf{1}-p^{\ell} \gamma$ and $\beta=\mathbf{1}-p^{\ell} \delta$ be two elements of $\mathbf{1}+p^{\ell} \mathrm{M}_{\mathrm{m}}\left(\mathbf{Z} / p^{n} \mathbf{Z}\right)$. Then

$$
\begin{aligned}
\alpha \beta \alpha^{-1} \beta^{-1} & =\left(\mathbf{1}-p^{\ell} \gamma\right)\left(\mathbf{1}-p^{\ell} \delta\right)\left(\mathbf{1}+p^{\ell} \gamma+\cdots+p^{n \ell} \gamma^{n}\right)\left(\mathbf{1}+p^{\ell} \delta+\cdots+p^{n \ell} \delta^{n}\right) \\
& =\mathbf{1}+p^{2 \ell}(\gamma \delta-\delta \gamma)+p^{3 \ell}(\cdots)+\cdots,
\end{aligned}
$$

so $\mathrm{PC}_{\mathrm{m}}(\ell)^{\prime}$ is included in $\mathrm{SL}_{\mathrm{m}}\left(\mathbf{Z} / p^{n} \mathbf{Z}\right) \cap \mathrm{PC}_{\mathrm{m}}(2 \ell)$. Conversely, consider the two elementary transvections $\sigma=\mathbf{1}+p^{\ell} e_{12}$ and $\tau=\mathbf{1}+p^{\ell} e_{21}$. One has

$$
\begin{aligned}
\sigma \tau \sigma^{-1} \tau^{-1} & =\left(\mathbf{1}+p^{\ell} e_{12}\right)\left(\mathbf{1}+p^{\ell} e_{21}\right)\left(\mathbf{1}-p^{\ell} e_{12}\right)\left(\mathbf{1}-p^{\ell} e_{21}\right) \\
& =\mathbf{1}+p^{2 \ell} e_{11}-p^{2 \ell} e_{22}-p^{3 \ell} e_{12}+p^{3 \ell} e_{21} .
\end{aligned}
$$


It follows that $\left(\mathrm{SL}_{\mathrm{m}}\left(\mathbf{Z} / p^{n} \mathbf{Z}\right) \cap \mathrm{PC}_{\mathrm{m}}(\ell)\right)^{\prime}$ contains an element that lies in $\mathrm{PC}_{\mathrm{m}}(2 \ell) \backslash \mathrm{PC}_{\mathrm{m}}(2 \ell+1)$. As $\left(\mathrm{SL}_{\mathrm{m}}\left(\mathbf{Z} / p^{n} \mathbf{Z}\right) \cap \mathrm{PC}_{\mathrm{m}}(\ell)\right)^{\prime}$ is a characteristic subgroup of $\mathrm{SL}_{\mathrm{m}}\left(\mathbf{Z} / p^{n} \mathbf{Z}\right) \cap \mathrm{PC}_{\mathrm{m}}(\ell)$, it is normal in $\mathrm{GL}_{\mathrm{m}}\left(\mathbf{Z} / p^{n} \mathbf{Z}\right)$. By Theorem 5, $\left(\mathrm{SL}_{\mathrm{m}}\left(\mathbf{Z} / p^{n} \mathbf{Z}\right) \cap \mathrm{PC}_{\mathrm{m}}(\ell)\right)^{\prime}$ contains $\mathrm{SL}_{\mathrm{m}}\left(\mathbf{Z} / p^{n} \mathbf{Z}\right) \cap \mathrm{PC}_{\mathrm{m}}(2 \ell)$. We have thus shown that for every natural number $k$, the $k$ th derived subgroup of $\mathrm{PC}_{\mathrm{m}}(\ell)$ is

$$
\mathrm{PC}_{\mathrm{m}}(\ell)^{(k)}=\mathrm{SL}_{\mathrm{m}}\left(\mathbf{Z} / p^{n} \mathbf{Z}\right) \cap \mathrm{PC}_{\mathrm{m}}\left(2^{\mathrm{k}} \ell\right) .
$$

The derived length of $\mathrm{PC}_{\mathrm{m}}(\ell)$ is the least $k$ such that $2^{k} \ell \geqslant n$.

Lemma 8. For natural numbers $k$ and $n \geqslant k^{2}+k$, let $\ell_{n}(k)=1+\left\lfloor\frac{n}{k+1}\right\rfloor$. Then $\ell_{n}(k)$ is the smallest natural number satisfying the equality $\left\lfloor\frac{n}{\ell_{n}(k)}\right\rfloor=k$.

Proof. Let $n=q(k+1)+r$ be the Euclidian division of $n$ by $k+1$, with $q \geqslant k$ and $0 \leqslant r<k+1$. Then one has

$$
0<\frac{k+1-r}{1+q} \leqslant 1 \text { hence }\left\lfloor\frac{n}{\ell_{n}(k)}\right\rfloor=\left\lfloor\frac{(k+1) q+r}{1+q}\right\rfloor=\left\lfloor k+1-\frac{k+1-r}{1+q}\right\rfloor=k,
$$

so $\ell_{n}(k)$ satisfies the equality. It is the smallest such, as one has

$$
\left\lfloor\frac{n}{\ell_{n}(k)-1}\right\rfloor=\left\lfloor\frac{n}{\left\lfloor\frac{n}{k+1}\right\rfloor}\right\rfloor=\left\lfloor\frac{n}{q}\right\rfloor=\left\lfloor k+1+\frac{r}{q}\right\rfloor \geqslant k+1 .
$$

Lemma 9. For natural numbers $k \geqslant 1$ and $n \geqslant 2^{k}$, let $d_{n}(k)=\left\lceil\frac{n}{2^{k}}\right\rceil$. Then $d_{n}(k)$ is the smallest natural number satisfying the equality $\left\lceil\log _{2} \frac{n}{d_{n}(k)}\right\rceil=k$.

Proof. One has

$$
\frac{n}{2^{k}} \leqslant\left\lceil\frac{n}{2^{k}}\right\rceil<\frac{n}{2^{k}}+1
$$

hence

$$
k-1 \leqslant k-\log _{2}\left(1+\frac{2^{k}}{n}\right)<\log _{2}\left(\frac{n}{\left\lceil\frac{n}{\left.2^{k}\right\rceil}\right.}\right) \leqslant k,
$$

so that $d_{n}(k)$ satisfies the equality. It is the smallest such, as

$$
\left\lceil\log _{2}\left(\frac{n}{\left\lceil\frac{n}{2^{k}}\right\rceil-1}\right)\right\rceil=\left\lceil\log _{2}\left(\frac{2^{k}}{\frac{2^{k}}{n}\left\lceil\frac{n}{2^{k}}\right\rceil-\frac{2^{k}}{n}}\right)\right\rceil=\left\lceil k-\log _{2}\left(\frac{2^{k}}{n}\left\lceil\frac{n}{2^{k}}\right\rceil-\frac{2^{k}}{n}\right)\right\rceil \geqslant k+1 .
$$

\section{RAdicAls OF $G$}

We now condiser

$$
G=\prod_{n \in \mathbf{N}} \mathrm{GL}_{\mathrm{m}}\left(\mathbf{Z} / p^{n} \mathbf{Z}\right) / \mathcal{U}
$$

We call Fitting subgroup of $G$ and write $F(G)$ the subgroup generated by all its normal nilpotent subgroups. By Zorn's Lemma, any nilpotent subgroup of $G$ of nilpotency class $k$ is contained in a maximal such, which might not be unique. 
Lemma 10. There is a first order formula $\varphi_{k}$ in the language of groups such that, for any group $N, N$ is nilpotent of class $k$ if and only if $N \models \varphi_{k}$.

Proof. Consider the formula

$\forall x_{1} \cdots \forall x_{k}\left[x_{1},\left[x_{2},\left[\cdots,\left[x_{k-1}, x_{k}\right] \cdots\right]\right]\right]=1 \wedge \exists y_{1} \cdots \exists y_{k-1}\left[y_{1},\left[y_{2},\left[\cdots,\left[y_{k-2}, y_{k-1}\right] \cdots\right]\right]\right] \neq 1$.

Theorem 11 (Łos). Let $\left(M_{i}\right)_{i \in \mathbf{N}}$ be a collection of L-structure, $\mathcal{U}$ an ultrafilter on $\mathbf{N}$ and $M$ the ultraproduct $\prod_{i} M_{i} / \mathcal{U}$. One has $M \models \varphi$ if and only if $\left\{i \in \mathbf{N}: M_{i} \models \varphi\right\}$ is in $\mathcal{U}$.

Theorem 12 (Fitting subgroup of $G$ ). If the ultrafilter $\mathcal{U}$ is non-principal, for every natural number $k, G$ has a unique maximal normal nilpotent subgroup $N_{k}$ of nilpotency class $k$

$$
N_{k}=\prod_{n \in \mathbf{N}}\left(\left(\mathbf{Z} / p^{n} \mathbf{Z}\right)^{\times} \cdot \mathbf{1}+p^{1+\left\lfloor\frac{n-1}{k+1}\right\rfloor} \mathrm{M}_{\mathrm{m}}\left(\mathbf{Z} / p^{n} \mathbf{Z}\right)\right) / \mathcal{U}
$$

hence the Fitting subgroup of $G$ is

$$
F(G)=\bigcup_{k=1}^{\infty} N_{k} .
$$

$F(G)$ is neither nilpotent, nor definable.

Proof. Let $k$ be a fixed natural number. By Lemma 6 and Lemma 8, the normal subgroup

$$
\left(\mathbf{Z} / p^{n} \mathbf{Z}\right)^{\times} \cdot \mathbf{1}+p^{1+\left\lfloor\frac{n-1}{k+1}\right\rfloor} \mathrm{M}_{\mathrm{m}}\left(\mathbf{Z} / p^{n} \mathbf{Z}\right)
$$

of $\mathrm{GL}_{\mathrm{m}}\left(\mathbf{Z} / p^{n} \mathbf{Z}\right)$ has nilpotency class $k$ for all but finitely many $n$. As $\mathcal{U}$ contains the Fréchet filter and as being of nilpotency class $k$ is expressible by a first order formula in the pure language of groups according to Lemma 10, by Łos Theorem, the ultraproduct

$$
\prod_{n \in \mathbf{N}}\left(\left(\mathbf{Z} / p^{n} \mathbf{Z}\right)^{\times} \cdot \mathbf{1}+p^{1+\left\lfloor\frac{n-1}{k+1}\right\rfloor} \mathrm{M}_{\mathrm{m}}\left(\mathbf{Z} / p^{n} \mathbf{Z}\right)\right) / \mathcal{U}
$$

is a normal nilpotent subgroup of class $k$ of $G$. Reciprocally, if $g$ belongs to a normal nilpotent subgroup of class $k$, then $g^{G}$ generates a normal nilpotent subgroup of class at most $k$. By Eos Theorem, there is a set $I \in \mathcal{U}$ such that for all $n \in I$, the conjugacy class $g_{n}^{G_{n}}$ generates a nilpotent normal subgroup $\left\langle g_{n}^{G_{n}}\right\rangle$ of $G_{n}$ of class at most $k$. Let $n \in I$ be fixed. By Theorem 5 , there is a unique natural number $1 \leqslant \ell \leqslant n$ such that

$$
\mathrm{SL}_{\mathrm{m}}\left(\mathbf{Z} / p^{n} \mathbf{Z}\right) \cap\left(\mathbf{1}+p^{\ell} \mathrm{M}_{\mathrm{m}}\left(\mathbf{Z} / p^{n} \mathbf{Z}\right)\right) \subset\left\langle g_{n}^{G_{n}}\right\rangle \subset\left(\mathbf{Z} / p^{n} \mathbf{Z}\right)^{\times} \cdot \mathbf{1}+p^{\ell} \mathrm{M}_{\mathrm{m}}\left(\mathbf{Z} / p^{n} \mathbf{Z}\right) .
$$

As $\mathbf{1}+p^{\ell} \mathrm{M}_{\mathrm{m}}\left(\mathbf{Z} / p^{n} \mathbf{Z}\right)$ is nilpotent of class $\left\lfloor\frac{n-1}{\ell}\right\rfloor$, one must have $k \geqslant\left\lfloor\frac{n-1}{\ell}\right\rfloor$. From Lemma 8 , it follows that $\ell \geqslant \ell_{n-1}(k)$ for all but finitely many $n$ in $I$, so that $g$ belongs to the desired ultraproduct.

To show that the Fitting subgroup of $G$ is not definable, let $g_{n, \ell}$ be the elementary transvection $1+p^{\ell} e_{i j}$ of $H_{n}$ for every $1 \leqslant \ell<n$. By Proposition 4 , one has

$$
\left\langle g_{n, \ell}^{G_{n}}\right\rangle=\mathrm{SL}_{\mathrm{m}}\left(\mathbf{Z} / p^{n} \mathbf{Z}\right) \cap\left(\mathbf{1}+p^{\ell} \mathrm{M}_{\mathrm{m}}\left(\mathbf{Z} / p^{n} \mathbf{Z}\right)\right)
$$

hence

$$
Z_{q}\left(\left\langle g_{n, \ell}^{G_{n}}\right\rangle\right)=\mathrm{SL}_{\mathrm{m}}\left(\mathbf{Z} / p^{n} \mathbf{Z}\right) \cap Z_{q}\left(\mathbf{1}+p^{\ell} \mathrm{M}_{\mathrm{m}}\left(\mathbf{Z} / p^{n} \mathbf{Z}\right)\right)
$$


so $g_{n, \ell}^{G_{n}}$ generates a nilpotent subgroup of nilpotency class $\left\lfloor\frac{n}{\ell}\right\rfloor$. For every $\ell \geqslant 1$, let $n=$ $k_{n} \ell+r_{n}$ where $k_{n}$ and $r_{n}$ denote the quotient and rest of the Euclidian division of $n$ by $\ell$ and let $g_{\ell}$ denote the class modulo $\mathcal{U}$ of

$$
\left(1_{\mathrm{GL}_{\mathrm{m}}(\mathbf{Z} / \mathbf{Z})}, 1_{\mathrm{GL}_{\mathrm{m}}(\mathbf{Z} / p \mathbf{Z})}, \ldots, 1_{\mathrm{GL}_{\mathrm{m}}\left(\mathbf{Z} / p^{\ell} \mathbf{Z}\right)}, g_{\ell+1,1}, g_{\ell+2,1}, \ldots, g_{2 \ell, 2}, g_{2 \ell+1,2}, \ldots, g_{n, k_{n}}, \ldots\right) .
$$

As $\left\lfloor\frac{n}{k_{n}}\right\rfloor=\ell$ holds for every $n \geqslant \ell^{2}$, the normal closure $\left\langle g_{\ell}\right\rangle^{G}$ is nilpotent of nilpotency class $\ell$ for every $\ell \geqslant 1$. Note that $G$ is $\aleph_{1}$-saturated by [Kei10, Theorem 5.6]. By [OH13, Theorem 1.3], the Fitting subgroup of $G$ is not definable.

Lemma 13. There is a first order formula $\varphi_{\ell}$ in the language of groups such that, for any group $S, S$ is soluble of derived length $\ell$ if and only if $S \models \varphi_{\ell}$.

Proof. Consider the term $t_{\ell}\left(x_{1}, \ldots, x_{2^{\ell}}\right)$ defined inductively by setting $t_{1}\left(x_{1}, x_{2}\right)$ to $\left[x_{1}, x_{2}\right]$ and $t_{\ell+1}\left(x_{1}, \ldots, x_{2^{\ell+1}}\right)$ to $\left[t_{\ell}\left(x_{1}, \ldots, x_{2^{\ell}}\right), t_{\ell}\left(x_{2^{\ell}+1}, \ldots, x_{2^{\ell+1}}\right)\right]$. Then consider the formula

$$
\forall x_{1} \cdots \forall x_{2^{\ell}} t_{\ell}\left(x_{1}, \ldots, x_{2^{\ell}}\right)=1 \wedge \exists y_{1} \cdots \exists y_{2^{\ell-1}} t_{\ell-1}\left(y_{1}, \ldots, y_{2^{\ell-1}}\right) \neq 1
$$

We call soluble radical of $G$ and write $R(G)$ the subgroup generated by all its normal soluble subgroups.

Theorem 14 (soluble radical of $G$ ). If the ultrafilter $\mathcal{U}$ is non-principal, for every natural number $\ell, G$ has a unique maximal normal soluble subgroup $S_{\ell}$ of derived length $\ell$

$$
S_{\ell}=\prod_{n \in \mathbf{N}}\left(\left(\mathbf{Z} / p^{n} \mathbf{Z}\right)^{\times} \cdot \mathbf{1}+p^{\left\lceil\frac{n}{2^{\ell}}\right\rceil} \mathrm{M}_{\mathrm{m}}\left(\mathbf{Z} / p^{n} \mathbf{Z}\right)\right) / \mathcal{U}
$$

hence the soluble radical of $G$ is

$$
R(G)=\bigcup_{\ell=1}^{\infty} S_{\ell}=F(G) .
$$

$R(G)$ is neither soluble, nor definable.

Proof. By Lemma 7, Lemma 9, Lemma 13 and Łos Theorem, $S_{\ell}$ is a normal soluble subgroup of $G$ of derived length $\ell$. By Theorem 5 and Lemma 9, $S_{\ell}$ is maximal such. Note that $1+\left\lfloor\frac{n}{2^{\ell}+1}\right\rfloor \leqslant\left\lceil\frac{n}{2^{\ell}}\right\rceil$ holds for every $n$ and $\ell$, so that one has $S_{\ell} \subset N_{2^{\ell}}$ hence $R(G)$ and $F(G)$ coincide.

\section{REFERENCES}

[AB14] Tuna Altmel and Paul Baginski, Definable envelopes of nilpotent subgroups of groups with chain conditions on centralizers, 2014.

[Bau90] Andreas Baudisch, On superstable groups, J. London Math. Soc. (2) 42 (1990), no. 3, 452-464.

[Bre38] Joel Brenner, The linear homogeneous group, Ann. of Math. (2) 39 (1938), no. 2, 472-493.

[Die55] Jean Dieudonné, La géométrie des groupes classiques, Ergebnisse der Mathematik und ihrer Grenzgebiete (N.F.), Heft 5, Springer-Verlag, Berlin, 1955.

[DW97] Jamshid Derakhshan and Frank O. Wagner, Nilpotency in groups with chain conditions, Quart. J. Math. Oxford Ser. (2) 48 (1997), no. 192, 453-466. 
[Kei10] H. Jerome Keisler, The ultraproduct construction, Ultrafilters across mathematics, Contemp. Math., vol. 530, Amer. Math. Soc., Providence, RI, 2010, pp. 163-179.

[Kli60] Wilhelm Klingenberg, Linear groups over local rings, Bull. Amer. Math. Soc. 66 (1960), 294-296.

[Mat93] Larry Matthews, The independence property in unstable algebraic structures I: p-adically closed fields, 1993.

[MT12] Dugald Macpherson and Katrin Tent, Pseudofinite groups with NIP theory and definability in finite simple groups, Groups and model theory, Contemp. Math., vol. 576, Amer. Math. Soc., Providence, RI, 2012, pp. 255-267.

[OH13] Abderezak Ould Houcine, A remark on the definability of the Fitting subgroup and the soluble radical, MLQ Math. Log. Q. 59 (2013), no. 1-2, 62-65.

[Wag95] Frank O. Wagner, The Fitting subgroup of a stable group, J. Algebra 174 (1995), no. 2, 599-609.

[Wag99] _ Nilpotency in groups with the minimal condition on centralizers, J. Algebra 217 (1999), no. $2,448-460$.

UNIVERSITÄT KONSTANZ

Fachbereich Matematik und Statistik

78457 Konstanz, Germany

(current address) PÔle de MAThÉmATiques de L'INSA DE Lyon, BÂtiment LÉonard de Vinci - 21, aVenue Jean Capelle, 69621 Villeurbanne, France

E-mail address: cedric.milliet@insa-lyon.fr 